\title{
The Residual Stress Simulation Analysis of the Vacuum Eutectic Soldering for Metal and LTCC Substrate
}

\author{
Chuanliang Wei ${ }^{\text {a }}$, Dejian Zhou ${ }^{\text {b, * }}$ \\ Electromechanical Engineering College, Guilin University of Electronic Technology, Guilin 541004, \\ China \\ achuan.liang@163.com, bemezdj@guet.edu.cn
}

Keywords: LTCC Substrate; Residual Stress; Numerical Simulation; Thermal-Structure Coupling

\begin{abstract}
A numerical simulation analysis model of the Metal/LTCC (Low Temperature Co-fired Ceramic) substrate vacuum eutectic soldering was built, through the study for the vacuum eutectic soldering process. Then making a thermal-structure coupling analysis for this model to research the residual stress distribution, and discuss the pressure, plating thickness and cooling rate impact for the soldering residual stress. Calculations indicate that residual stress at the interface between heterogeneous materials is bigger, and solder angular position, where induce the interface crack easily, exists stress concentration, due to the metal and LTCC substrate material mechanics performance does not match. Cooling rate, plating thickness and pressure have influence on the residual stress, and this conclusion have certain reference significance for optimizing and improving the Metal/LTCC substrate vacuum eutectic soldering process.
\end{abstract}

\section{Introduction}

LTCC substrate is widely used in the manufacture of high density integration components (Multi-Chip Module and System level packaging etc.) and Millimeter microwave devices thanks to its very good electrical and mechanical properties, which include low dielectric constant, high wiring layer, and small wiring conductor sheet resistance as well as possibility of embedding passive device components. It has good thermal conductivity and stability. In the manufacture of a radio frequency module, a metal carrier and a metal ring is soldered on the LTCC substrate to improve the substrate performance and system reliability. The research find that the residual stress induced by thermal mismatch will influence the interconnection precision, even induce interface cracks and eventually lead to system failure [1, 2]. This paper uses large finite element analysis software ANSYS, makes a numerical simulation analysis for Metal/LTCC substrate vacuum eutectic soldering based on the thermal-structure coupling analysis method. Discussing the pressure, plating thickness and cooling rate impact for the soldering residual stress, and providing certain theoretical basis to optimize and improve the Metal/LTCC substrate vacuum eutectic soldering process.

\section{Metal/LTCC Substrate Vacuum Eutectic Soldering Process Analysis}

The simulation conditions have to be defined according technological process to build the Metal/LTCC substrate numerical simulation model. The Figure 1 describe the temperature curve of the Metal/LTCC substrate vacuum eutectic soldering process. We can learn that the vacuum eutectic soldering process have for stages which include preprocess, preheating, soldering and cooling [3]. During preprocess, the furnace is been vacuumed again and again to eliminate the residual gas in the furnace. Preheating stage includes warming-preheating temperature, which works as buffer function to make the device surface temperature uniformity. The soldering stage includes warming-soldering temperature, the temperature reach soldering temperature and solder began to melt, solder coating with metal and LTCC substrate in full. The cooling stage is the temperature-fall period, the solder is cooling solidification with the temperature drop, and the soldering being completed finally. 

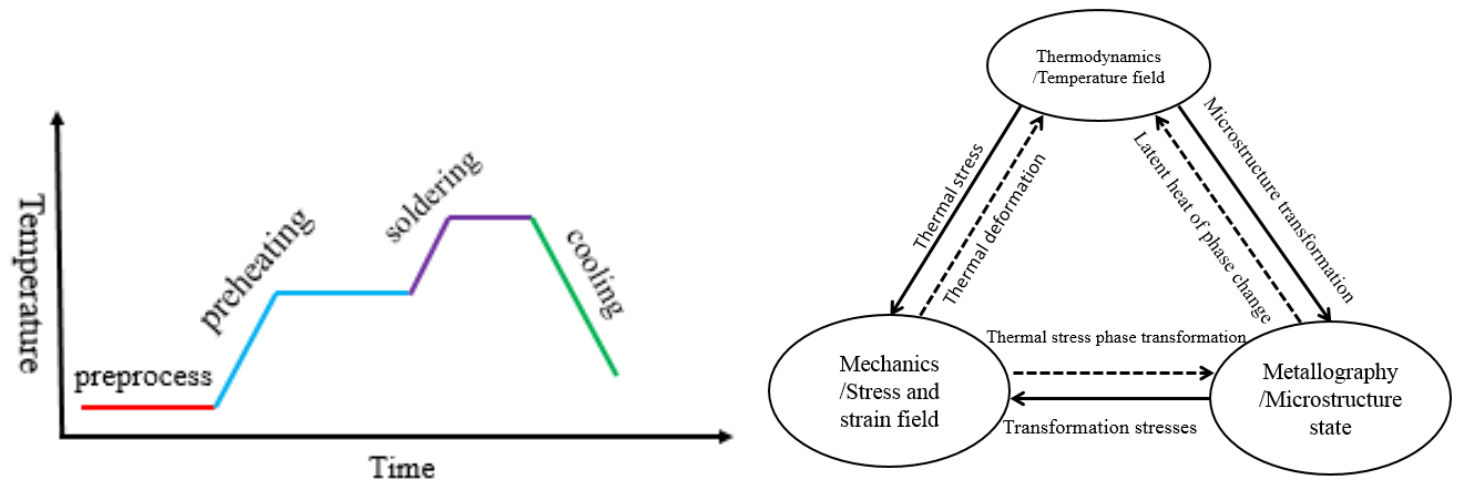

Fig.1 Temperature curve Fig. 2 Process decomposition \& coupling relationship

Soldering is a complex process, which include physics, heat transfer, metallurgy and mechanics. Soldering is decomposed into temperature field, stress and strain, and microstructure state [4] according fundamental process. This decomposition is very meaningful for numerical analysis, especially the soldering residual stress and soldering deformation. The coupling relationship between them is shown in Figure 2. The dotted line indicate relationship is weak, which can be ignored in the engineering [5].

We mainly think about the temperature field and microstructure influence to the stress-strain, while ignore the stress-strain influence to them. So this paper uses the order coupled method to analysis the temperature influence to the stress-strain, and use the element deactivation/reactivation to simulation the state change during the cooling stage.

\section{The Creation of the Numerical Model}

The study object is a simplify structure which is constituted by the Metal, soldering, plating metal and LTCC substrate. As shown in Figure 3. The size of the metal base is $65 \mathrm{mmx} 25 \mathrm{mmx} 3 \mathrm{~mm}$. The size of the AuSn is $65 \mathrm{mmx} 25 \mathrm{mmx} 3 \mathrm{~mm}$. The size of the Au is $65 \mathrm{mmx} 25 \mathrm{mmx} 3 \mathrm{~mm}$. The size of the LTCC base is $65 \mathrm{mmx} 25 \mathrm{mmx} 3 \mathrm{~mm}$. The material physical parameters [6, 7] is shown in table 1.

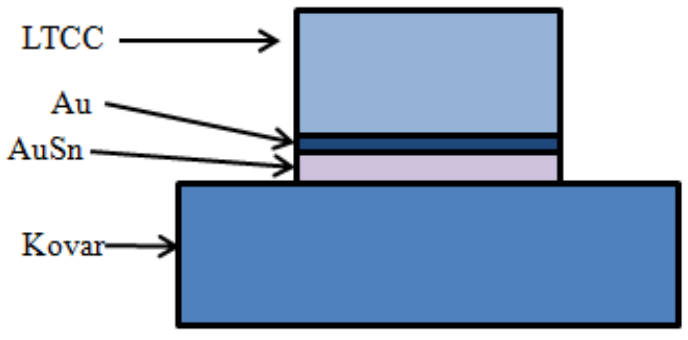

Fig 3 Metal and LTCC substrate assembly diagram

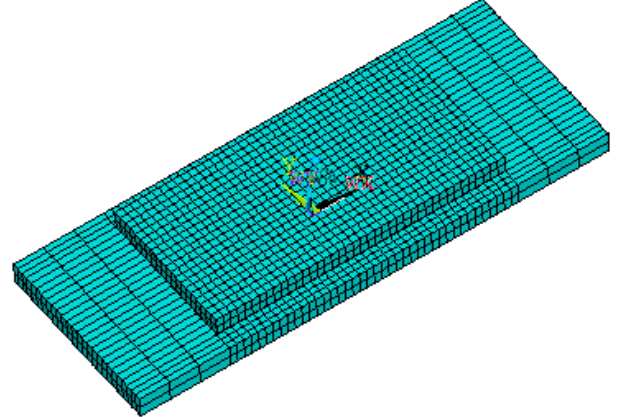

Fig.4 Meshing FE

Set up the finite element analysis geometric model according the geometric structure parameters, and meshing the geometric model using the hexahedral mesh. The meshing is shown in Figure 4.

Metal, LTCC substrate and solder are assembled and clamped by clamp during the vacuum eutectic welding. Then put them on graphite heating plate in the vacuum furnace to heat. There are several assumptions in the numerical model: 1.The vacuum eutectic solder furnace vacuum degree is so high enough that there is no natural convection. 2. Do not think about the dissolution and diffusion of the solder to base mental because they have little effect on residual stress. We can know from hypothesis 1 that there is mainly thermal radiation and heat conduction for the transfer of energy and have no thermal convection. So we mainly think the thermal radiation during the thermal analysis. In addition, we do not create the model of clamp, but converting to constraint condition of the finite element model. That is, fixing the $\mathrm{Z}$ direction of the bottom of the finite element model. Fixing the $\mathrm{X}$, $\mathrm{Y}$ direction of the around of the finite element model. Putting 600Pa uniform pressure on the top of the finite element model. 
Table 1 material property parameters

\begin{tabular}{ccccccc}
\hline Material & $\begin{array}{c}\text { Youngs } \\
\text { Modulus(GPa) }\end{array}$ & $\begin{array}{c}\text { Poisson } \\
\text { Ratio }\end{array}$ & $\begin{array}{c}\text { Thermal } \\
\text { cond. }[\mathrm{w} /(\mathrm{m} \cdot \mathrm{k})]\end{array}$ & $\begin{array}{c}\text { TCE } \\
\left(\mathrm{ppm} /{ }^{\circ} \mathrm{C}\right)\end{array}$ & $\begin{array}{c}\text { Density } \\
\left(\mathrm{kg} / \mathrm{m}^{3}\right)\end{array}$ & $\begin{array}{c}\text { Specific } \\
\text { Heat } \\
{[\mathrm{J} /(\mathrm{Kg} \cdot \mathrm{k})]}\end{array}$ \\
\hline LTCC & 82 & 0.26 & 3 & 5.5625 & 3100 & 350 \\
Au & 70 & 0.44 & 317 & 14.2 & 19320 & 126 \\
Au80Sn20 & 68 & 0.405 & 58 & 16 & 14520 & 380 \\
Kovar & 140 & 0.317 & 17.3 & 5.5 & 3100 & 439 \\
\hline
\end{tabular}

\section{Simulation Analysis Result}

We can know from the Figure 5 and Figure 6 that the angle position stress of the interface between metal and LTCC is maximum and there is stress concentration. So it is the position where Metal and LTCC substrate components easy to have a cracking. This is because the CTE (Coefficient of Thermal Expansion) mismatch result in larger residual stress in the Solder edge and angle.
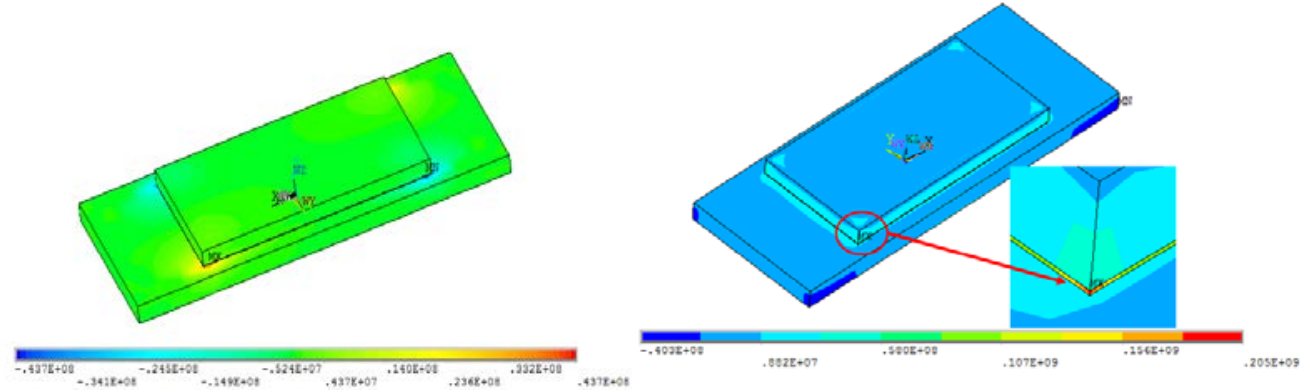

Fig.5 Shear residual stress distribution Fig.6 Equivalent residual stress distribution

Now, drawing the solder location stress distribution curve respectively along the path A-B and C-D, as shown in Figure7. From the distribution of the curve, we find that no matter path A-B or path C-D, the stress distribution of solder is that the edge stress is larger and complex distribution, but the stress is lesser in the central position.
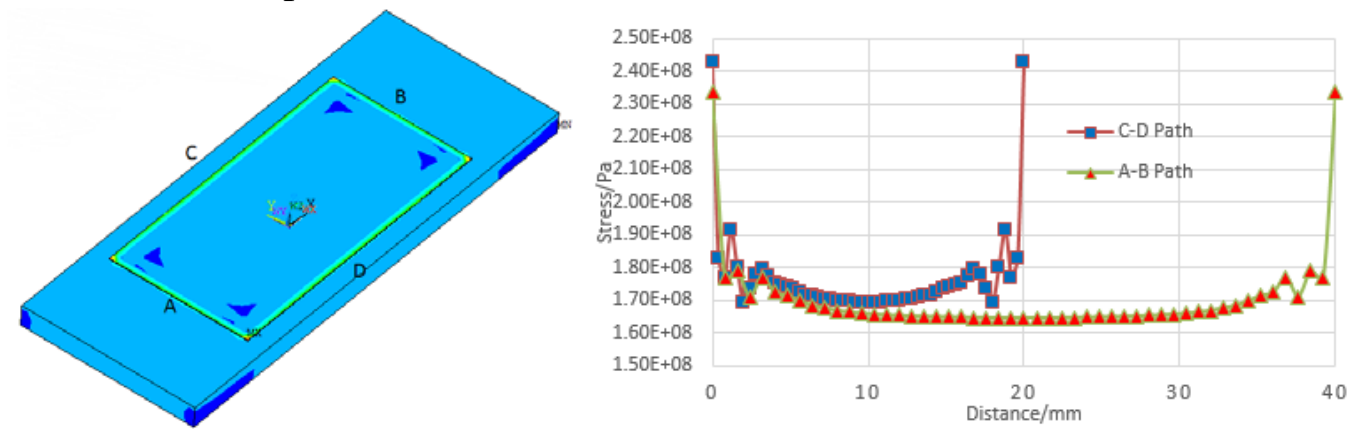

Fig.7 Residual stress distribution after soldering

Getting the residual stress in the solder corner nodes and drawing the change of residual stress with time curve, according the residual stress distribution, shown in Figure8. From the curve we can know that the stress is lesser at the beginning of the cooling. Solder gradually solidified and stress increase gradually until stabilization with the time goes on. The reason of the stress change is caused by mainly interaction between solder and substrate. They produce stress because the different shrinkage which caused by CTE mismatch. Plastic deformation will appear when the stress is bigger than the yield limitation with the increase of stress.

\section{Influence Factors}

Metal and structure of LTCC substrate volume is relatively small, so the residual stress is more sensitive for some process parameters and size. So we should optimize these parameters to reduce the residual stress in the practical engineering. This paper discuss the pressure, plating thickness and cooling rate impact for the soldering residual stress by the numerical simulation analysis. 
The Effects of Cooling Rate. Figure 9 shown the equivalent residual stress change curve the corners of the solder under different cooling rate with the case of other conditions unchanged. From the curve we can know that vacuum eutectic solder of the equivalent stress decreases after soldering with the increase of cooling rate. This is because faster cooling rate can accelerate the solidification of solder, and reduce the deformation produced by different solder solidification time.

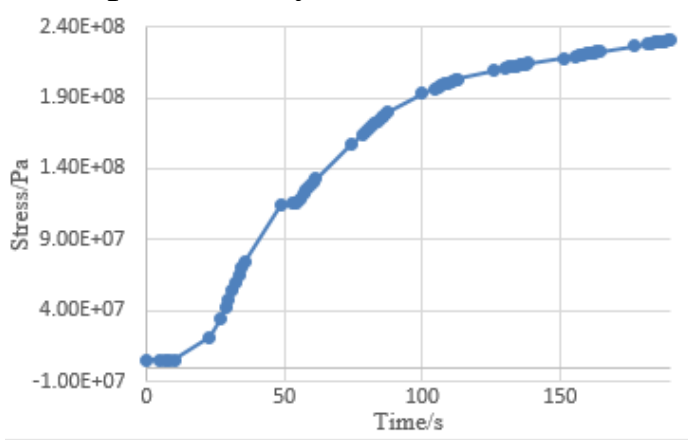

Fig.8 Node residual stress VS time

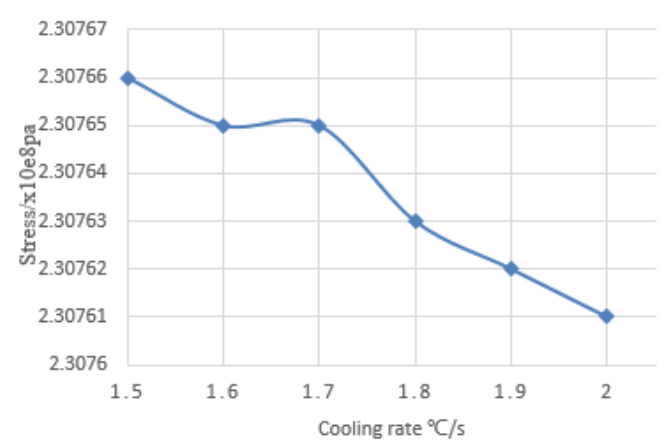

Fig.9 Cooling rate VS residual stress

The Influence of Coating Thickness. Changing the coating thickness with other sizes unchanged. Choosing 0,10,20,30 and $40 \mu \mathrm{m}$ as the coating thickness to make the numerical simulation analysis in the range of 0 to $40 \mu \mathrm{m}$. Extracting equivalent residual stress in the solder corners after soldering, and drawing the curve, shown as Figure 10. From this curve we find that the stress decreases after soldering with the increase of coating thickness. Because the plating metal, as a kind of intermediate material, its CTE is middle between solder and substrate, so it has certain slowing effect for the residual stress after soldering.

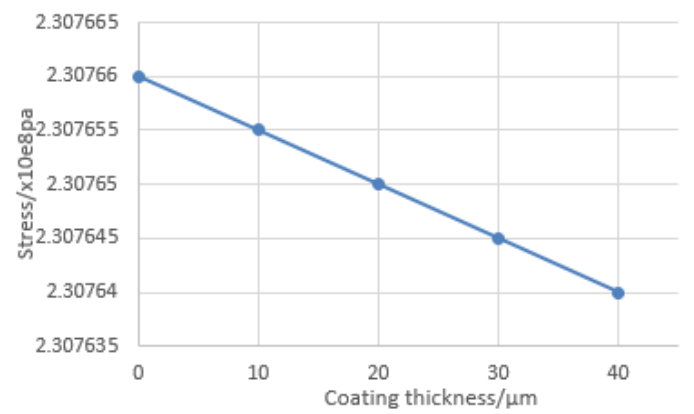

Fig.10 Coating thickness VS residual stress

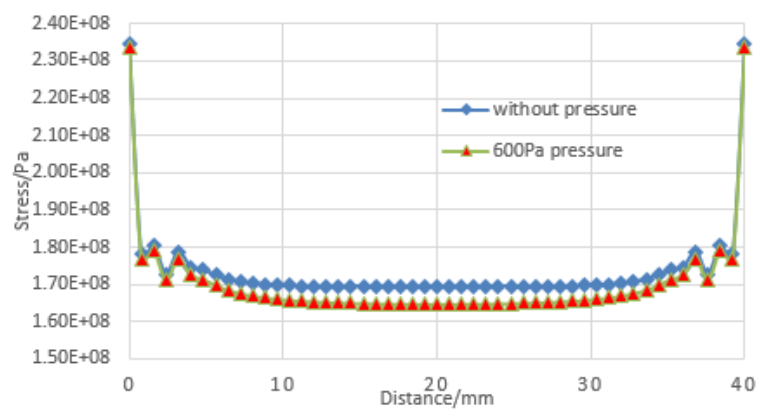

Fig.11 Pressure VS residual stress 
The Influence of Pressure. Figure 11 shows the A-B residual stress under distribution of the path after soldering without pressure load and $600 \mathrm{~Pa}$ pressure load cases. From the diagram we know that residual stress is rescued after pressure on the model. Because compressive stress and residual stress of soldering components are in the opposite direction and it offsets part of the residual stress. This is in agreement with the results of the related study [8]. But the press loaded on the model should be in appropriate range in order to avoid too bigger pressure damaging the whole soldering components

\section{Result}

This paper uses large finite element analysis software ANSYS, makes a numerical simulation analysis for Metal/LTCC substrate vacuum eutectic soldering based on the thermal-structure coupling analysis method. Getting the residual stress distribution, and discuss the pressure, plating thickness and cooling rate impact for the soldering residual stress. Calculations indicate that residual stress at the interface between heterogeneous materials is bigger, and solder angular position, where induce the interface crack easily, exists stress concentration, due to the metal and LTCC substrate material mechanics performance does not match. Increase the cooling rate and coating thickness can reduce the residual stress, on some degree. Pressure can offset part of the residual stress after soldering. This conclusion have certain reference significance for the optimizing and improving the Metal/LTCC substrate vacuum eutectic soldering process. But more accurate material performance parameter and more in-depth research is also needed, if we want to get more accurate residual stress analysis results during the Metal and LTCC substrate vacuum eutectic soldering process.

\section{Acknowledgement}

This work was supported by the National Defense Basic Scientific Research "Multiple Energy Loading Mechanism Analysis" and "Function Layer Interface Matching Rule and Multiple Energy Coordinated Control Method”.

\section{Reference}

[1] Zhang, J. X, Chandel, R.S, Effect of residual stress on the strength of an alumina-steel joint by partialtransient liquid phase (PTLP) brazing, Journal of Materials Processing Technology. 2000(12):220-225.

[2] Charles, Y. Identification d'un mode devieillissement dans un assemblagecéramique-métal [D]. E-cole Normale Supérieure de Cachan, 2002.

[3] Xie Fei, Liu Mei Yao, Vacuum eutectic technology research and application [J]. Electronics Process Technology, 2006(6):344-347.

[4] Wang Janhua, Welding numerical simulation technology and its application [M]. Shanghai Jiao Tong University Press, 2003(Version 1).

[5] Cheng Shuli, The numerical analysis of soldering residual stress based on the temperature and stress field [D]. Nanchang University, master's degree thesis, 2007.

[6] J.Lechkkeri, Thermo-mechanical Simulations of LTCC packages for RF MEMS Applications [J]. Mechanical and Multiphysics Simulation and Esperiment in Micro-Electronicss and Micro- Systems, EuroSimE2010.

[7] Http://www.alpharep.de/downloads/ATWALL\%20Kovar\%20www.Alpharep

[8] Lei Yongping, the numerical analysis for ceramic-metal brazing residual stress [J]. Transactions of the china welding institution, 2003(5):33-36 